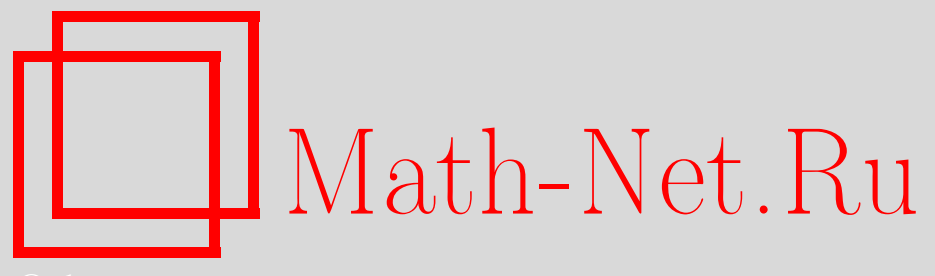

А. А. Грушо, Е. Е. Тимонина, Оценка времени, требуемого для организации скрытого канала, Дискрет. матем., 2003, том 15, выпуск 2, 40-46

DOI: https://doi.org/10.4213/dm191

Использование Общероссийского математического портала Math-Net.Ru подразумевает, что вы прочитали и согласны с пользовательским соглашением http://www. mathnet.ru/rus/agreement

Параметры загрузки:

IP: 3.95 .254 .165

26 апреля 2023 г., 17:24:58

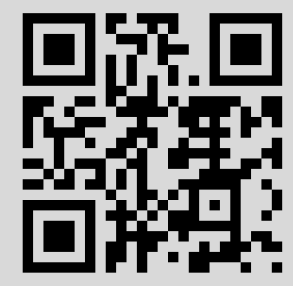




\title{
Оценка времени, требуемого для организации скрытого канала
}

\author{
๑ 2003 г. А. А. Грушо, Е. Е. Тимонина
}

\begin{abstract}
В работе построена математическая модель скрытого канала взаимодействия агентов в глобальной сети и в закрытом сегменте локальной сети. Канал передает информацию через устройство защиты, работающее по принципу IPsec с использованием инкапсулирования и шифрования пакетов. В работе анализируется асимптотика времени обучения агента языку передачи данных. Доказано, что если число $n$ узлов в произвольном сегменте и число $m$ сегментов стремятся к бесконечности, то при определенных соотношениях параметров время обучения есть $O\left(m^{2} n \ln n\right)$.

Работа выполнена при поддержке Российского фонда фундаментальных исследований, проект 01-01-00895.
\end{abstract}

Целью данной работы является демонстрация возможностей скрытого управления агентами в защищенной вычислительной среде в условиях, когда при построении защиты слабо учитываются угрозы существования скрытых каналов [2, 5]. Данная работа не содержит описания всех возможных скрытых каналов для управления агентами в защищенной среде, мы лишь приводим математические модели, в рамках которых доказывается возможность существования таких каналов в различных ситуациях, и изучаем различные свойства таких каналов.

Построению скрытых каналов в среде Интернет посвящен целый ряд работ. Наиболее близкой к нашим целям является работа [3]. В ней показано, что с помощью упорядочивания адресов потока пакетов в Интернет трафике можно построить скрытый канал с высокой пропускной способностью $\left(\log _{2} k\right.$ !, где $k$ - длины упорядоченных цепочек пакетов). В отличие от [3] нас интересует возможность преодоления устройств защиты, помещенных между закрытым сегментом локальной сети и глобальной сетью (например, Интернет).

Пусть имеется $m+1$ сегментов локальных вычислительных сетей, $S_{0}, S_{1}, \ldots, S_{m}$, в каждом из которых имеются рабочие станции со своими локальными адресами и шлюзы, соединяющие локальные сети с глобальной сетью (например, Интернет). Пусть $s_{0}, s_{1}, \ldots, s_{m}$ - адреса шлюзов сегментов локальных вычислительных сетей $S_{0}, S_{1}, \ldots, S_{m}$, которые представляют эти сегменты в глобальной сети. Мы считаем, что для общения между собой рабочие станции различных сегментов используют виртуальную частную сеть (VPN), которая работает следующим образом. Пакет от рабочей станции с адресом $x$ в сегменте $S_{i}$ должен быть передан на рабочую станцию с адресом $y$ в сегменте $S_{j}$. Пакет передается следующим образом:

- от машины с адресом $x$ к машине $\mathrm{PC}(i, 1)$ в сегменте $S_{i}$; 
- далее пакет попадает на узел защиты УЗ $(i)$, в котором пакет шифруется и инкапсулируется в пакет (пакеты) с адресом отправителя $s_{i}$ и адресом получателя $s_{j}$, пакеты имеют одинаковую длину и другие одинаковые параметры;

- инкапсулированный пакет из УЗ $(i)$ попадает на хост глобальной сети $\mathrm{PC}(i, 2)$, который направляет его через глобальную сеть на аналогичный хост $\mathrm{PC}(j, 2)$;

- далее пакет направляется на узел защиты УЗ $(j)$, на выходе которого восстанавливается исходный пакет, посланный от $x$ к $y$, этот пакет поступает на $\mathrm{PC}(j, 1)$;

- PC $(j, 1)$ отправляет пакет к абоненту у в сегмент $S_{j}$.

Предполагается, что в глобальной сети и в каждом сегменте $S_{0}, S_{1}, \ldots, S_{m}$ имеются программно-аппаратные агенты нарушителя безопасности, которые для выполнения своих враждебных функций должны получать инструкция от нарушителя из глобальной сети (НГС). Будем считать, что нарушитель глобальной сети полностью контролирует компьютеры $\mathrm{PC}(j, 2), j=0,1, \ldots, m$. Программно-аппаратные агенты нарушителя внутри сегментов локальной сети $S_{0}, S_{1}, \ldots, S_{m}$ контролируют соответственно компютеры РС $(j, 1), j=0,1, \ldots, m$. Мы считаем, что узлы защиты УЗ $(i)$ сделаны правильно, так что никто из нарушителей не может контролировать эти узлы. Таким образом, управление программно-аппаратным агентом в любом из сегментов со стороны нарушителя из глобальной сети связано с построением канала связи от $\operatorname{PC}(j, 2)$ к $\operatorname{PC}(j, 1)$. Утечка информации связана с построением канала от $\operatorname{PC}(j, 1)$ к $\operatorname{PC}(j, 2)$. Представляет интерес построение таких способов передачи данных через Интернет, которые позволяют передавать скрытые сообщения через маршрутизаторы сети.

Все машины сегмента локальной сети не знают адреса $s_{0}, s_{1}, \ldots, s_{m}$. Также ни один из хостов глобальной сети (в частности, все машины $\mathrm{PC}(j, 2), j=0,1, \ldots, m)$ не знает внутренние адреса сегментов. В силу того, что шифрование и вычисление кодов аутентификации происходит на узлах защиты, нарушитель не может построить канал связи взаимодействия с программно-аппаратным агентом сегмента, используя шифртекст или служебные атрибуты пакетов. Таким образом, мы предполагаем, что единственными зависимыми параметрами, известными на $\mathrm{PC}(j, 2)$ и $\mathrm{PC}(j, 1)$ для входного потока пакетов, являются адреса отправителя пакетов. При передаче пакетов от $\operatorname{PC}(j, 1)$ к $\operatorname{PC}(j, 2)$ единственными параметрами являются адреса получателей пакетов. Эта зависимость выражается в виде функции $s=f(a)$, которая отображает множество $\{a\}$ внутренних адресов каждого сегмента на внешний адрес $s$ соответствующего шлюза. Всего у сегмента $S_{0}$ возможно $S_{1} \cup \ldots \cup S_{m}$ адресов, $M=\sum_{i=1}^{m}\left|S_{i}\right|$, где $\left|S_{i}\right|$ - число элементов в множестве $S_{i}, i=1, \ldots, m$.

Рассмотрим задачу построения сообщений, которые могут активизировать агента в PC $(0,1)$. Для этого необходимо передать полубит информации, который может быть распознан отслеживающей системой агента в $\mathrm{PC}(0,1)$. Простейший способ состоит в следующем. Множество $S=\left\{s_{1}, \ldots, s_{m}\right\}$ делим на две части $S(1)$ и $S(2)$ таким образом, что интенсивности входных потоков от отправителей из $S(1)$ приблизительно совпадают с интенсивностью потока от отправителей из $S(2)$. Пусть РС $(0,2)$ посылает пакеты на УЗ(0) по очереди из $S(1)$ и $S(2)$. Отслеживающая система агента в РС $(0,1)$ вычисляет расстояния между пакетами, приходящими из одного адреса независимо от получателя пакетов. Если пакеты в УЗ(0) посылаются по указанному выше правилу, то все вычисленные расстояния - четные (за исключением ошибок, связанных с вставкой или потерей пакетов). Если мы предполагаем, что ошибок мало, то превышение числа четных рассто- 
яний заданного порога может рассматриваться, как указание агенту активизироваться и начать следующий этап получения указаний от нарушителя в глобальной сети.

Второй этап передачи информации от нарушителя из глобальной сети к своему агенту в $\mathrm{PC}(0,1)$ состоит в передаче натурального числа $k \geqslant 2$. Передача числа $k$ происходит аналогично запуску программно-аппаратного агента в $\mathrm{PC}(0,1)$. В этом случае все адреса делятся на $k$ классов, приблизительно совпадающие по суммарной интенсивности. Передача приходящих пакетов происходит таким образом, чтобы расстояние между одинаковыми адресами $s$ было кратно $k$.

Третий этап передачи информации от нарушителя из глобальной сети к своему агенту в $\mathrm{PC}(0,1)$ состоит в обучении. Мы считаем, что множество $S$ линейно упорядочено так, что $s_{1}<s_{2}<\ldots<s_{m}$. Процедура обучения необходима для того, чтобы максимально полно восстановить на РС $(0,1)$ порядок $\leqslant$ на множестве $f^{-1}(S)$. При этом мы считаем, что если $x$ и $y$ принадлежат $f^{-1}(s)$, то $x=y$. Процедура обучения состоит в следующем. $\mathrm{PC}(0,2)$, получив очередные $k$ пакетов с адресами $s_{i_{1}}, s_{i_{2}}, \ldots, s_{i_{k}}$, упорядочивает их в соответствии с отношением $\leqslant$ (меньше либо равно) и посылает данные пакеты на узел защиты для последующей передачи $\mathrm{PC}(0,1)$. Мы считаем, что $\mathrm{PC}(0,1)$ обладает большими вычислительными возможностями для того, чтобы в реальном масштабе времени проводить сопоставление полученных данных и восстанавливать порядок $\leqslant$ на $f^{-1}(S)$. Время обучения - это число упорядоченных наборов из $k$ адресов каждый, которые нужно передать для восстановления в РС $(0,1)$ информации об упорядочении адресов из множества $S$ в такой степени, чтобы можно было с большой степенью уверенности читать информацию, передаваемую от $\mathrm{PC}(0,2)$ к $\mathrm{PC}(0,1)$, то есть за это время порядок должен быть восстановлен в той степени, чтобы можно было передавать информацию упорядоченными $k$-группами адресов.

На четвертом этапе происходит передача информации с помощью кодирования 1 возрастающей последовательностью $k$ адресов, которые формируются на $\mathrm{PC}(0,2)$, и кодирования 0 убывающей последовательностью $k$ адресов, которые формируются на $\mathrm{PC}(0,2)$. В том случае, если комбинация очередных $k$ адресов состоит из одного адреса $s$, данная последовательность передается в любом порядке и не несет никакой информации. Аналогично, комбинации одинаковых адресов $s$ в очередных $k$ пакетах между собой не упорядочиваются. Передача начинается с посылки векторов из 0, после этого может быть использован любой, например, префиксный код, начинающийся с 1. Передача заканчивается последовательностью из 1 , которая продолжается до посылки следующего сообщения.

Оценим время $N$ обучения программного агента в $\mathrm{PC}(0,1)$ согласно процедуре третьего этапа, описанного выше. Формализуем и упростим задачу следующим образом. Будем считать, что все исходные адреса из $S$ представляются независимо друг от друга и равновероятно. Пусть $k=2$. Ясно, что при $k>2$ время обучения не превосходит это время для $k=2$. Для простоты считаем, что мощности всех сегментов равны: $\left|S_{1}\right|=\ldots=\left|S_{m}\right|=n$. Будем называть упорядоченные пары при обучении дугами, тогда на множестве $S=S_{1} \cup \ldots \cup S_{m}$ агент $\mathrm{PC}(0,1)$ видит случайный граф $G$ на $M$ вершинах с $N$ ориентированными дугами. Дуги появляются независимо друг от друга, и вероятность каждой дуги равна $\left(1 / n^{2}\right)\left(m+\left(\begin{array}{c}m \\ 2\end{array}\right)\right)$. Обозначим через $G / S_{i}, i=1, \ldots, m$, ограничение графа $G$ на множество $S_{i}$ вершин, $i=1, \ldots, m$.

Теорема 1. Пусть $n, m \rightarrow \infty$ так, что $m=O\left(n^{\delta}\right)$, где $0<\delta<1 / 2$. Тогда существует константа $b>0$ такая, что при $N \geqslant b^{2} n \ln n$ вероятность восстановления порядка стремится $\kappa 1$. 
Для доказательства теоремы потребуются следующие леммы.

Лемма 1. Пусть графы $G / S_{i}, \quad i=1, \ldots, m$, сильно связны и для каждого $i=1, \ldots, m-1$ существует, по крайней мере, одна дуга, соединяющая вершину из множества $S_{i}$ с вершиной из множества $S_{i+1}$. Тогда порядок $\leqslant$ на множестве $S$ восстанавливается полностью.

Доказательство. Для любой пары $(x, y), x \leqslant y$, по условиям леммы в графе $G$ существует ориентированный путь из $x$ в $y$. Транзитивное замыкание этого пути восстанавливает отношение $x \leqslant y$, что и требовалось доказать.

При $i=1, \ldots, m$ рассмотрим случайные графы $G / S_{i}(p)$ на $n$ различных вершинах, в которых дуги появляются независимо друг от друга с вероятностью $p$.

Лемма 2. Если $n, m \rightarrow \infty$ так, что $m=O\left(n^{\delta}\right)$, где $0<\delta<1 / 2$, то существует такое $b>0$, что при $p \geqslant b \ln n / n$ вероятность того, что все графы $G / S_{i}(p), i=1, \ldots, m$, сильно связны, не меньше, чем $n^{-\varepsilon}$ для некоторого $\varepsilon>0$.

Доказательство. Для доказательства леммы фиксируем произвольную пару вершин $(x, y)$ из $S_{i}$ и оценим вероятность достижимости из $x$ вершины $y$ в графе $G / S_{i}(p)$. Для этого рассмотрим следующий способ построения графа $G / S_{i}(p)$. Сначала построим граф $G /\left(S_{i} \backslash\{y\}\right)(p)$ на множестве $S_{i} \backslash\{y\}$. Затем независимо друг от друга построим дуги, соединяющие множество $S_{i} \backslash\{y\}$ и $y$ при условии, что граф на множестве $S_{i} \backslash\{y\}$ фиксирован. Из оценок в работе [4] следует, что для любого фиксированного $x$ при $p \geqslant \alpha /(n-1)$, $\alpha>0$, в графе $G / S_{i}(p)$ для больших $n$ с вероятностью, не меньшей $1-K \theta^{n}$ для некоторых $K>0,0<\theta<1$, существует сn при некотором $c, 0<c<1$, достижимых из $x$ вершин. Тогда условная вероятность того, что из заданного набора достижимых в графе $G /\left(S_{i} \backslash\{y\}\right)(p)$ из $x$ вершин нет ни одной дуги в $y$, асимптотически не превосходит $(1-p)^{c n}$. Выбором $b$ при $p=b \ln n / n$ мы можем сделать эту вероятность асимптотически не большей, чем $n^{-3}$. В силу независимости появления дуг вероятность недостижимости из вершины $x$ вершины $y$ не будет превосходить $K \theta^{n}+n^{-3}$. Тогда вероятность того, что в графе $G / S_{i}(p)$ существует по крайней мере одна пара $(x, y)$, для которой $y$ не достижим из $x$, асимптотически не превосходит $1 / n$. Отсюда следует, что вероятность того, что хотя бы один граф из $G / S_{i}(p), i=1, \ldots, m$, асимптотически не является связным, не превосходит $n^{-1+\delta}$. Лемма доказана.

Пусть $N_{i}, i=1, \ldots, m,-$ число дуг из $N$ в графе $G$, которые попали на множество $S_{i} ; N_{i j}, i<j$, - число дуг, которые соединяют множества $S_{i}$ и $S_{j}$. Рассмотрим условное распределение ограничения графа $G$ на множества $S_{1}, \ldots, S_{m}$ при фиксированных $N_{1}, \ldots, N_{m}$. Тогда на каждом множестве $S_{i}$ индуцируется случайный ориентированный мультиграф с петлями с $N_{i}$ дугами, причем все $N_{i}$ дуг попадают на каждое из возможных мест независимо друг от друга с вероятностью $1 / n^{2}$. Рассмотрим отображение $\varphi$ множества всех мультиграфов $\left\{G^{\prime}\right\}$ на $n$ вершинах с $N_{i}$ дугами в множество ориентированных графов на тех же вершинах без петель и параллельных дуг, которое определяется следующим образом. В образе $\varphi\left(G^{\prime}\right)$ есть дуга $(x, y)$ тогда и только тогда, когда в графе $G^{\prime}$ есть хотя бы одна дуга $(x, y)$, а петли исключаются. Если при случайном выборе $G^{\prime}$ из множества $\left\{G^{\prime}\right\}$ каждая дуга может попасть равновероятно на любое из $n^{2}$ мест независимо друг от друга, то функщия $\varphi$ порождает на соответствующем множестве графов вероятностное распределение. Это вероятностное распределение обладает следующими свойствами. 
(1) Все дуги в графе $\varphi\left(G^{\prime}\right)$ появляются независимо друг от друга. В самом деле, появление дуги $(x, y)$ в графе $\varphi\left(G^{\prime}\right)$ определяется только дугами $(x, y)$ в графе $G^{\prime}$ и, тем самым, не зависит от появления всех остальных дуг в графе $G^{\prime}$. Следовательно, появление дуги $(x, y)$ в графе $\varphi\left(G^{\prime}\right)$ не зависит от появления других дуг в графе $\varphi\left(G^{\prime}\right)$.

(2) Все дуги в графе $\varphi\left(G^{\prime}\right)$ появляются с одинаковой вероятностью $p$, которая определяется из соотношения

$$
1-p=\left(1-1 / n^{2}\right)^{N_{i}}
$$

Если $N_{i} \geqslant b n \ln n$, то $p \geqslant b \ln n / n$.

(3) Если граф $\varphi\left(G^{\prime}\right)$ сильно связен, то граф $G^{\prime}$ также сильно связен. В самом деле, из существования ориентированного пути от вершины $x$ к вершине $y$ в графе $\varphi\left(G^{\prime}\right)$ следует, что такой же путь существует в графе $G^{\prime}$.

Таким образом, если $N_{i} \geqslant b n \ln n$, то из леммы 2 следует, что граф $\varphi\left(G^{\prime}\right)$ с вероятностью, стремящейся к 1 , сильно связен. И если все $N_{i} \geqslant b n \ln n$, то условные распределения при условиях $\left\{N_{i}\right\}$ таковы, что при этих распределениях вероятность сильной связности всех графов $G / S_{i}, i=1, \ldots, m$, стремится к 1 .

Лемма 3. Если $N, n, m \rightarrow \infty$ так, что $m=O\left(n^{\delta}\right)$, где $0<\delta<1 / 2$, то существует такое $C>0$, что при $N /\left(m^{2} n \ln n\right) \geqslant C$ с вероятностью, стремящейся $\kappa 1, N_{i} /(n \ln n) \geqslant b$ для всех $i=1, \ldots, m$.

Доказательство. В условиях, когда $\left|S_{i}\right|=n, i=1, \ldots, m$, случайные величины $\left\{N_{i}, N_{i j}\right\}$ распределены как частоты в равновероятной схеме размещения $N$ различимых дробинок (дуг) в $m+\left(\begin{array}{c}m \\ 2\end{array}\right)$ различных ящиков (соответствующих множествам $S_{i}$ и парам $\left(S_{i}, S_{j}\right)$ ). Это следует из того, что для каждого таким образом определенного ящика существует ровно $n^{2}$ возможных дуг, каждая из которых в исходной схеме появляется с вероятностью $1 / n^{2}$. Тогда по неравенству Чебышева

$$
\mathbf{P}\left(\bigcup_{i=1}^{m}\left(\left|N_{i}-N /\left(m+\left(\begin{array}{c}
m \\
2
\end{array}\right)\right)\right|>\varepsilon \sqrt{N}\right)\right) \leqslant \frac{m N\left(1-1 /\left(m+\left(\begin{array}{c}
m \\
2
\end{array}\right)\right)\right)}{\left(m+\left(\begin{array}{c}
m \\
2
\end{array}\right)\right) N \varepsilon^{2}}=O\left(\frac{1}{m \varepsilon^{2}}\right) .
$$

Отсюда следует, что в условиях леммы с вероятностью, стремящейся к 1 , для всех $i=1, \ldots, m$

$$
N_{i} \geqslant N /\left(\begin{array}{l}
m \\
2
\end{array}\right)-\varepsilon \sqrt{N} \geqslant \frac{3 N}{2 m^{2}} .
$$

Тогда при $N /\left(m^{2} n \ln n\right) \geqslant 2 b / 3$ из предыдущего неравенства получаем, что для всех $i=1, \ldots, m$ в асимптотических условиях леммы $N_{i} /(n \ln n) \geqslant b, i=1, \ldots, m$, с вероятностью, стремящихся к 1 . Лемма доказана.

Лемма 4. Если $N, n, m \rightarrow \infty$ так, что $m=O\left(n^{\delta}\right)$, где $0<\delta<1 / 2$, то при $N /\left(m^{2} n \ln n\right) \geqslant C, C>0$, с вероятностью, стремячейся $\kappa 1, N_{i, i+1} \geqslant 1$ для всех $i=1, \ldots, m-1$.

Доказательство. По неравенству Чебышева, как и в лемме 6,

$$
\mathbf{P}\left(\bigcup_{i=1}^{m-1}\left(\left|N_{i, i+1}-N /\left(m+\left(\begin{array}{c}
m \\
2
\end{array}\right)\right)\right|>\varepsilon \sqrt{N}\right)\right)=O\left(\frac{1}{m \varepsilon^{2}}\right) .
$$

Отсюда следует утверждение леммы. 
Объединяя полученные выше оценки, получаем утверждение теоремы.

Если $\mathrm{PC}(0,2)$ знает приблизительно число $M=|S|$, то он может оценить время обучения величиной $O(M \ln M)$.

Рассмотрим случай, когда $n \rightarrow \infty$, а $m$ фиксировано. Тогда имеет место следующая теорема.

Теорема 2. Если $n \rightarrow \infty, m$ фиксировано и отношение $N /(n \ln n) \geqslant C$ при некотором $C>0$, то с вероятностью, стремящейся $\kappa 1$, порядок $\leqslant$ восстанавливается.

Доказательство. Из теоремы Крамера [1] следует, что вектор случайных величин $\left(\ldots, N_{i}, \ldots, N_{i j}, \ldots\right)$ имеет асимптотически нормальное распределение. Из этого утверждения следуют необходимые асимптотические неравенства $N_{i} \geqslant b n \ln n$ и $N_{i, i+1} \geqslant 1$. Тогда при выполнении условий теоремы 2, используя результаты, полученные при доказательстве теоремы 1 , находим, что с вероятностью, стремящейся к 1 , графы $G / S_{i}$, $i=1, \ldots, m$, сильно связны и существует, по крайней мере, одна дуга, связывающая $S_{i}$ c $S_{i+1}, i=1, \ldots, m$. Теорема доказана.

Если агент в РС $(0,1)$ априори запрограммирован так, что всю посылаемую информацию (как при обучении, так и при передаче данных) он посылает обратно, то процесс обучения значительно сокращается. Этому способствует адаптация агентов РС $(0,2) \mathrm{k}$ полученным от $\mathrm{PC}(0,1)$ результатам обучения, или $\mathrm{PC}(0,1)$ дает знание, когда надо повторно передать байт. При наличии обратной связи, а также в том случае, когда после обучения агент в $\mathrm{PC}(0,2)$ передаст агенту в $\mathrm{PC}(0,1)$ программу пересылки информации из закрытого сегмента в глобальную сеть с помощью построенного языка, $\mathrm{PC}(0,1)$ может передавать через скрытый канал любую конфиденциальную информацию в глобальную сеть, минуя узел защиты.

Естественным желанием является построение защиты от данного канала с помощью вставки на случайные места неинформативных пакетов со случайными адресами, которые агенты не могут отличить от информативных. Однако, как показывает следующий пример, такую защиту можно преодолеть. Пусть $s_{1}, s_{2}$ - два возможных адреса отправителя, $k, n / 2<k<n$, адресов $s_{1}$ без вкраплений адреса $s_{2}$ считается единицей, а $k$, $n / 2<k<n$, адресов $s_{2}$ без вкраплений адреса $s_{1}$ считается нулем. Серии адресов $s_{1}$ или $s_{2}$ длины, меньшей $n / 2-1$, не воспринимаются как передаваемые символы. Пусть агенты в $\mathrm{PC}(0,1)$ и $\mathrm{PC}(0,2)$ знают этот язык, и пусть для простоты сообщения передаются из закрытого сегмента локальной сети от агента $\mathrm{PC}(0,1)$ через агента $\mathrm{PC}(0,2)$ в глобальную сеть. Остальные пакеты и генерируемые узлом защиты ложные пакеты располагаются в промежутках между пакетами с адресом $s_{1}$, когда передается вектор пакетов, соответствующий передаче 1 по скрытому каналу, или в промежутках между пакетами с адресом $s_{2}$, когда передается вектор пакетов, соответствующий передаче 0 по скрытому каналу, или в других местах. То есть, скрытой передачи нет, если после вычеркивания всех адресов, кроме адресов $s_{1}$ и $s_{2}$, нет серий адресов $s_{1}$ и $s_{2}$ длины, большей $n / 2$. Если такая серия длины, большей $n / 2$, из пакетов с адресами $s_{1}$ есть, то это означает передачу 1 по скрытому каналу. Аналогично, если есть серия длины, большей $n / 2$, из пакетов с адресами $s_{2}$, то это означает передачу 0 по скрытому каналу.

Пусть при передаче пакетов кроме пакетов с адресами, отличными от $s_{1}$ и $s_{2}$, случайно и равновероятно выбираются $r$ адресов, по которым посылаются ложные пакеты. Рассмотрим условия, при которых предложенная схема устойчива к случайным вставкам. Вероятность появления более одного раза пакета с адресом $s_{1}$ в серии пакетов с адресом $s_{2}$, соответствующей передаваемому по скрытому каналу 0 , равна $P$ и определяется из 
условия

$$
1-P=(1-1 / m)^{r}+(r / m)(1-1 / m)^{r-1} \geqslant 1-(r-1) r / m^{2} .
$$

Если отношение $r / m$ мало, то появление не более одной вставки пакета с адресом $s_{1}$ не снижает читаемость кода 0 при передаче по скрытому каналу, так как остается серия из пакетов с адресом $s_{2}$, длины, большей $n / 2$. Поскольку в этом случае мы предполагаем маловероятным появление более одной вставки пакетов с адресом $s_{1}$, появления ложных 0 в сообщении по скрытому каналу не будет.

Таким образом, случайные вставки $r$ ложных пакетов не исключают чтения сообщения по скрытому каналу.

\section{Список литературы}

1. Крамер Г., Математические методы статистики. Мир, Москва, 1975.

2. Тимонина Е. Е., Скрытые каналы (обзор). JetInfo (2002) №11, 3-11.

3. Ahsan K., Kundur D., Practical data hiding in TCP/IP. Workshop Multimedia and Security ACM Multimedia'02, Juan-les-Pins, French Riviera, 2002.

4. Ajtai M., Komlos J., Szemeredi E., The longest path in a random graph. Combinatorica (1981) 1, $1-12$.

5. A guide to understanding covert channel analysis of trusted systems NCSC TG-030. National Computer Security Center, Fort George G. Meade, Maryland 20755-6000, 1993.

Статья поступила 05.04.2003. 